\title{
Implementing a Metapopulation Bass Diffusion Model using the R Package deSolve
}

by Jim Duggan

\begin{abstract}
Diffusion is a fundamental process in physical, biological, social and economic settings. Consumer products often go viral, with sales driven by the word of mouth effect, as their adoption spreads through a population. The classic diffusion model used for product adoption is the Bass diffusion model, and this divides a population into two groups of people: potential adopters who are likely to adopt a product, and adopters who have purchased the product, and influence others to adopt. The Bass diffusion model is normally captured in an aggregate form, where no significant consumer differences are modeled. This paper extends the Bass model to capture a spatial perspective, using metapopulation equations from the field of infectious disease modeling. The paper's focus is on simulation of deterministic models by solving ordinary differential equations, and does not encompass parameter estimation. The metapopulation model in implemented in $\mathrm{R}$ using the deSolve package, and shows the potential of using the $\mathrm{R}$ framework to implement large-scale integral equation models, with applications in the field of marketing and consumer behaviour.
\end{abstract}

\section{Introduction}

Diffusion is a fundamental process in physical, biological, social and economic settings (Rahmandad and Sterman, 2008). In the business arena, consumer products frequently go viral by the word of mouth effect between consumers. In this scenario, products experience rapid sales growth, which eventually slows as the number of potential adopters decline. A classic dynamic model to capture these growth processes is the Bass model (Bass, 1969), which is based on the assumption that the timing of purchases is related to the number of previous buyers. This model provided good predictions of the sales peak, and the timing of the peak when compared to historical data. Providing model-based estimates of peak timing can assist with production capacity planning and sales distribution strategies, for example, to ensure that enough product items are available in time to match future demand. A representation of the Bass model is shown in Figure 1, and this captures the scenario whereby newer technologies are not immediately adopted by all potential buyers, and a diffusion process is set into motion (Norton and Bass, 1987). This version of the Bass model is informed by standard epidemiological diffusion models(Vynnycky and White, 2010), and so the term force of persuasion is analogous to the epidemiological term force of infection.

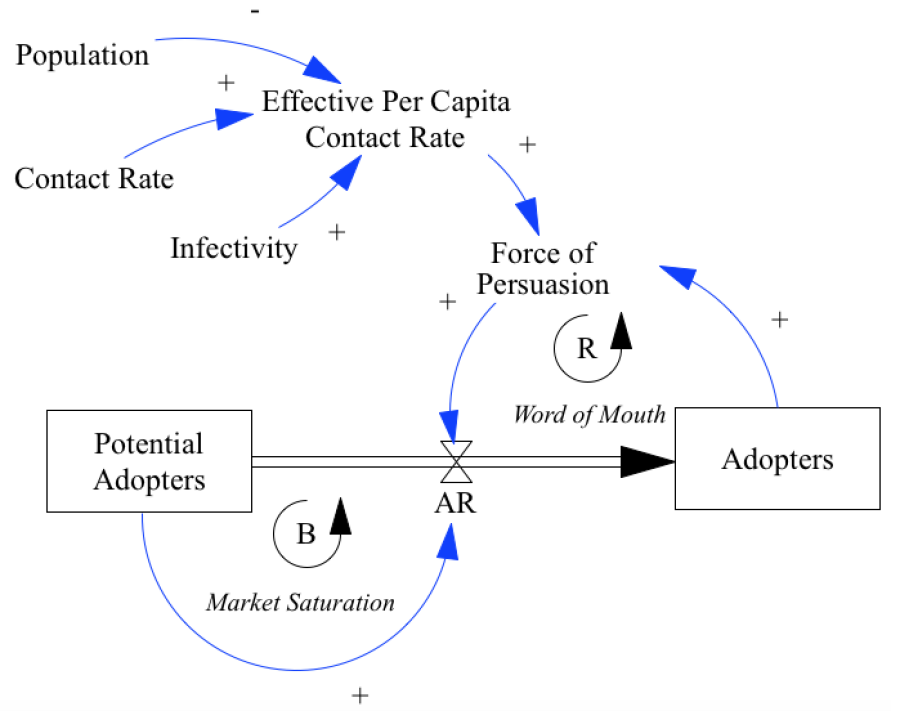

Figure 1: Market diffusion model, aggregate version. $A R$ represents the adoption rate (derivative), and + and - refer to positive and negative association respectively. The symbol $\boldsymbol{B}$ represents a negative feedback loop (balancing), while $\boldsymbol{R}$ indicates a positive feedback loop (reinforcing). 
The modeling methodology used in this paper is system dynamics (Sterman, 2000; Duggan, 2016), a method for modeling social systems, which focuses on how feedback structures (i.e. causal loops) impact overall system behavior. Feedback is a defining element of system dynamics (Lane, 2006), where a feedback loop is a chain of circular causal links represented in a model. There are two types of feedback which impact a variable: negative feedback which opposes the direction of change in a loop variable (for example, a theromstat controller that regulates heat in a room), whereas positive feedback amplifies a variable's value, as in the case of the contagion effect in infectious disease spread. At a technical level, system dynamics models are solved using integration, where integrals are referred to as stocks, and derivatives are modeled as flows. System dynamics diagrams are intuitive, as they present a dynamic system in terms of the stocks (containers), and flows (valves), which control the rate at which stocks fill and drain over time.

\section{Bass diffusion model}

The Bass diffusion model has two integrals: Potential Adopters (1), which model individuals in the population that have yet to acquire a product, and Adopters (2), which represent people who have purchased a product, and are in a position to influence others to initiate a purchase. For this paper, the initial values of these stocks are 99,999 and 1 respectively, giving a total population size of 100,000. These initial values will also be maintained across the metapopulation model, which provides a useful basis for comparing the outputs of both models.

$$
\begin{gathered}
\text { PotentialAdopters }=\int_{0}^{t}-A R d t \\
\text { Adopters }=\int_{0}^{t} A R d t
\end{gathered}
$$

The flow equation $A R(8)$ captures a number of concepts. First, there is the idea of the effective per capita contact rate, known by the parameter $\beta$ (3), which is based on:

- The contact rate (4) between members of the population, which is an estimate of how often people interact.

- The infectivity (5) of these interactions, which is a way of modeling how likely it is for an individual to be convinced to purchase, based on an interaction with an adopter.

- The total population (6), which is the denominator of the equation.

$$
\begin{gathered}
\beta=\frac{\text { ContactRate } \times \text { Infectivity }}{\text { Population }} \\
\text { ContactRate }=3 \\
\text { Infectivity }=0.15 \\
\text { Population }=100,000
\end{gathered}
$$

Given the value for $\beta$, it is then possible to calculate the force of persuasion $\rho$, which defines the proportion of potential adopters that will convert at each time period. This equation (7) is similar to the epidemiological term known as the attack rate, which determines how many susceptible people become infected per time period in classic infectious disease models (Anderson et al., 1991).

$$
\rho=\beta \times \text { Adopters }
$$

With the value for $\rho$ evaluated, the adoption rate is simply the product of this value with the number of potential adopters in the population, as shown in (8).

$$
A R=\rho \times \text { PotentialAdopters }
$$

These equations have three properties that add to the robustness of the model, These are:

- if there are no contacts in the population, $\beta$ will equal 0 , and no adoption will occur;

- if infectivity is zero, $\beta$ will also equal 0 , which will stop any adoption;

- if there are no adopters, then the force of persuasion will be zero, and no adoptions will occur. 
Equations (1)-(8) can be implemented using the deSolve package (Soetaert et al., 2010, 2016), which solves initial value problems written as ordinary differential equations (ODE), differential algebraic equations (DAE), and partial differential equations (PDE). For modeling diffusion processes, the R package EpiDynamics (Baquero and Marques, 2015) was also considered, however it does not currently provide functionality to solve two-compartment SI models, which forms the basis of the Bass model. The R package EpiModel (Jenness et al., 2016) also contains an API that is flexible to formulate a number deterministic compartmental models. For our scenario, the ODE solver in deSolve is used, where the differential equations are encapsulated in a function.

The initial segment of $\operatorname{code}^{1}$ loads the packages, sets the simulation start time, finish time, and time step, and constructs the appropriate time vector that is needed by deSolve. Following that, the two integrals (stocks) are created, along with their initial values, and auxiliary parameters initialised within a vector. In naming variables, Hungarian Notation ( $\mathrm{Li}$ and Prasad, 2005) is used, to distinguish between stocks (s), flows (f) and exogenous parameters (a), known in system dynamics as auxiliaries.

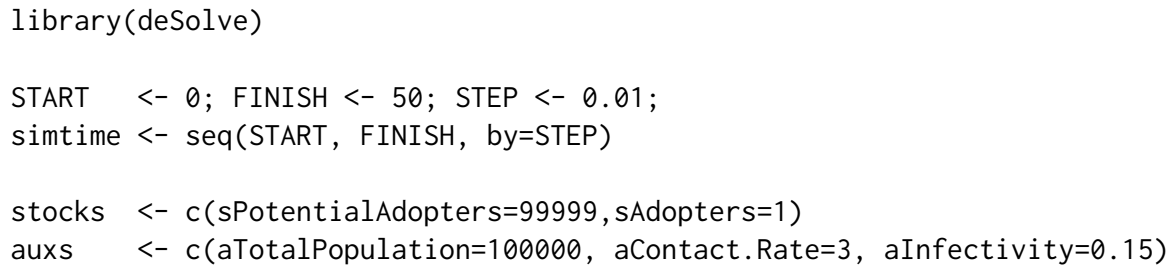

Next the function containing the model equations is constructed. This is called by the deSolve framework for every solution step of the ODE, and the parameters passed in include:

- The current simulation time,

- A vector containing all integrals (stocks) and their values,

- A vector containing the parameters used in the stock and flow model.

The $\mathrm{R}$ code for the function is shown below, and the corresponding model equations are also referred to.

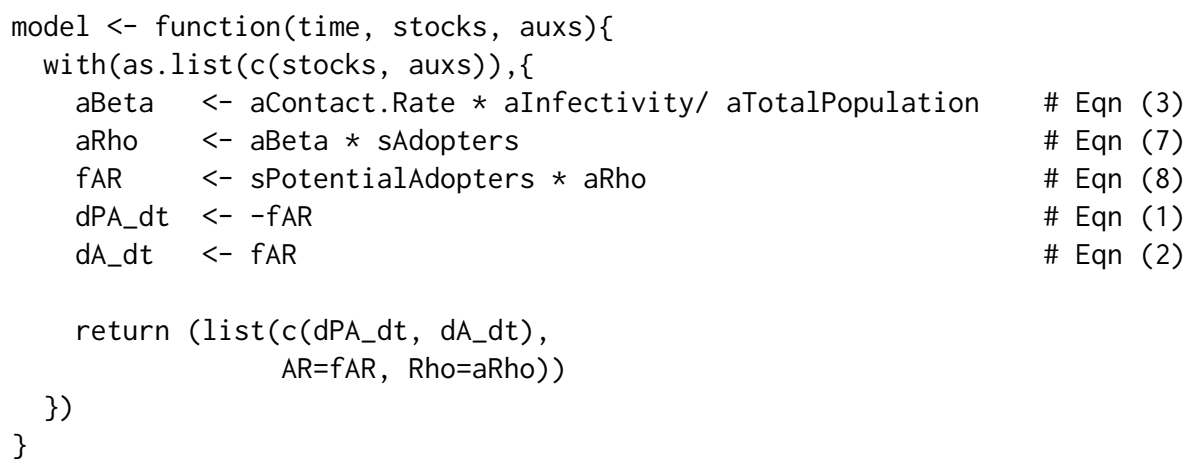

To run the simulation, the function ode() is called with five parameters, including the vector of stocks, the simulation time vector, the callback model function, any exogenous parameters (none in this case), and the numerical integration algorithm to use (Euler's method is deployed, as this is commonly used as an integration method for social systems (Sterman, 2000)). The results from ode() are then wrapped in a data frame, in order to make use of ggplot().

$0<-$ data.frame(ode(y=stocks, times=simtime, func $=$ model, parms=auxs, method="euler"))

Figure 2 shows the classic behavior of the Bass model, as the dynamics of the adopter exhibit s-shaped growth as the customer base saturates to its maximum possible value over time.

In summary, the aggregate model is useful to capture the overall dynamics of the diffusion process. However, for spatial diffusion models a revised equation structure is needed, and one such model based on the dynamics of infectious disease transmission - is now described.

\footnotetext{
${ }^{1}$ See supplemental material included with the paper
} 


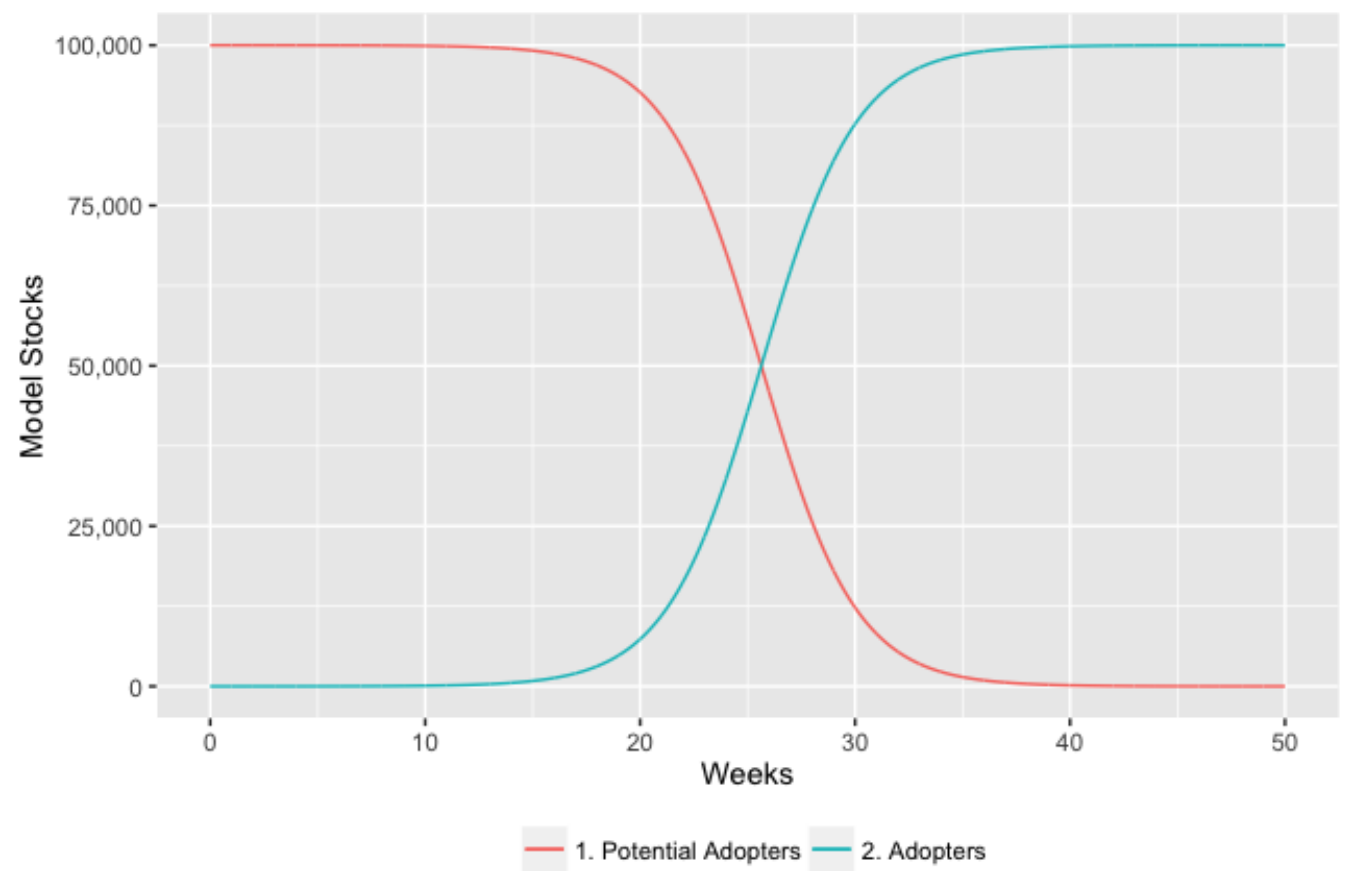

Figure 2: Simulation of the Bass model

\section{Metapopulation diffusion model}

Metapopulation structures are a powerful feature in dynamic modeling, as they facilitate the investigation of finer-grained behaviours and dynamics than can be achieved through an aggregate model. The metapopulation concept is to subdivide the entire population into distinct subpopulations, each with independent dynamics, and combine this with limited interaction between each subpopulation (Keeling and Rohani, 2008). The approach is appropriate if there are significant variations in model parameters across the population. For example, in disease dynamics, empirical studies show nonrandom mixing in populations, including data on the transmission of tuberculosis (Borgdorff et al., 1999), and contact patterns across Europe show highly assortative mixing patterns with age (Mossong et al., 2008). A similar argument can be made for product adoption models, and for this scenario, a regional spatial structure is proposed as the main mechanism for subdividing the model. Figure 3 shows a hypothetical regional structure, where the population centers (colours reflect population density) are in the middle of the region, and models an unbalanced regional development which is a feature of many countries.

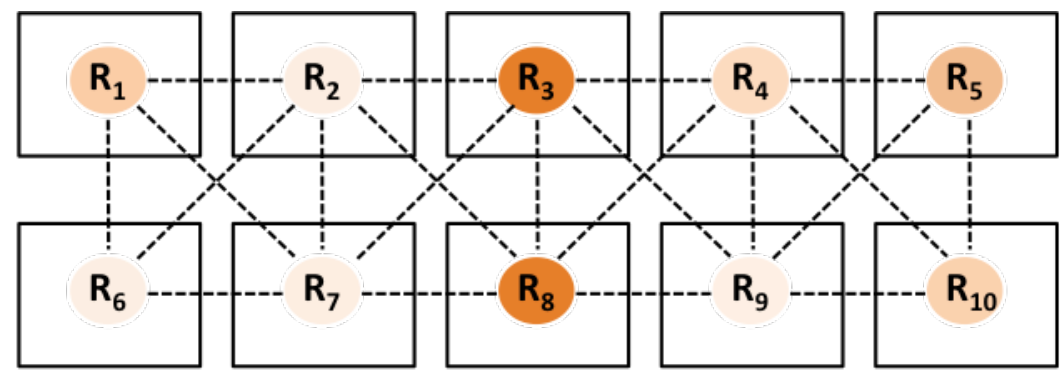

Figure 3: Spatial structure for metapopulation diffusion model

Out of an overall population of 100,000, the proportions in each region are captured in Table 1.

\begin{tabular}{cccccccccc}
\hline R1 & R2 & R3 & R4 & R5 & R6 & R7 & R8 & R9 & R10 \\
\hline $10 \%$ & $1 \%$ & $21 \%$ & $8 \%$ & $17 \%$ & $4 \%$ & $5 \%$ & $25 \%$ & $3 \%$ & $6 \%$ \\
\hline
\end{tabular}

Table 1: Population proportions by region 
At a structural level, the diffusion model has 20 stocks, based on the stock and flow structure in Figure 1. The integral equations (1) and (2) are replicated for each region, and each region has its own adoption rate (8). This adoption rate is formulated as the product of each region's force of persuasion and the number of adopters, as specified in (9).

$$
A R_{i}=\rho_{i} \times \text { Potential Adopters }_{i}
$$

The force of persuasion $\rho_{i}$ reflects the spatial properties of the model, and is a weighted sum of the per capita effective contact rates from region $j$ to region $i$, multiplied by the number of adopters $\left(A_{j}\right)$ in region $j$ (10). This structure is based on standard epidemiological transmission equations between cohorts, where the $j^{\text {th }}$ cohort is infectious (an adopter), and the $i^{\text {th }}$ cohort is susceptible (a potential adopter).

$$
\rho_{i}=\sum_{j=1}^{N} \beta_{i j} . A_{j}
$$

Equation (10) can also be conveniently expressed in matrix form, as shown in (11).

$$
\left(\begin{array}{c}
\rho_{1} \\
\rho_{2} \\
\vdots \\
\rho_{N}
\end{array}\right)=\left(\begin{array}{cccc}
\beta_{11} & \beta_{12} & \ldots & \beta_{1 N} \\
\beta_{21} & \beta_{22} & \ldots & \beta_{2 N} \\
\vdots & \vdots & \ddots & \vdots \\
\beta_{N 1} & \beta_{N 2} & \ldots & \beta_{N N}
\end{array}\right)\left(\begin{array}{c}
A_{1} \\
A_{2} \\
\vdots \\
A_{N}
\end{array}\right)
$$

The $\beta$ component, originally introduced in (3), is the effective contact rate per capita, and therefore the square matrix component of (11) can be further factored into (12) which shows the transmission calculations between each region. The denominator is the same for each matrix row, and is the population of each region containing the potential adopters.

$$
\beta_{i j}=e_{i j} / N_{i}
$$

The effective contact values $(e)$ are based on the contact rates between the different sectors. The contact rates are calculated from the normal contact rate for a region $(n)$, multiplied by a distancing weighting measure using a power law function (Brockmann et al., 2006), useful for individual-based models (Mungovan et al., 2011; Liu et al., 2012), given that these structures have also been successfully implemented in models of infectious disease (Meyer et al., 2014). This equation is shown in (13), and the distance is the Euclidean distance function that uses the grid coordinates as a reference (14), given the points $\left(x_{1}, y_{1}\right)$ and $\left(x_{2}, y_{2}\right)$.

$$
\begin{gathered}
c_{i j}=n_{j} \cdot\left(d_{i j}+1\right)^{-\alpha} \\
d_{i j}=\sqrt{\left(x_{2}-x_{1}\right)^{2}+\left(y_{2}-y_{1}\right)^{2}}
\end{gathered}
$$

To provide flexibility for experimentation, the parameter $\alpha$ (15) provides scope to moderate the contact rates between the different regions. With $\alpha=0$, the contact rates do not change from the original region to other regions, whereas higher values will cause contact rates to decrease as the distance between regions increases.

$$
\alpha \geq 0
$$

The effective contact rates $(e)$, which model the word of mouth effect from region to region is shown in (16), which is the product of the contact rates $(c)$ and infectivity $(f)$, which models the probability of transmission. This equation provides the necessary information to process the matrix specified in (11).

$$
e_{i j}=c_{i j} \cdot f_{j}
$$

Tables 2-5 show sample parameter values for the model. In Table 2, sample normal contact rates per area are shown, with R5 and R8 having the highest values, given that these have the highest population proportions. Table 3 summarises arbitrary infectivity values, which do not vary significantly from region to region, as an individual's ability to persuade others is not assumed to be dependent on the overall population density. Table 4 shows the distance values calculated between each region, where the coordinates are based on the row/column location of a region, shown earlier in Figure 3. Table 5 then shows the final contact rates, using (13), with $\alpha=1.0$, which shows that the contact rate falls as 


\begin{tabular}{cccccccccc}
\hline R1 & R2 & R3 & R4 & R5 & R6 & R7 & R8 & R9 & R10 \\
\hline 4.58 & 0.56 & 10.12 & 4.61 & 10.00 & 1.64 & 2.53 & 14.46 & 1.53 & 2.95 \\
\hline
\end{tabular}

Table 2: Sample normal contact rates $(n)$ per area, weighted by population density

\begin{tabular}{cccccccccc}
\hline R1 & R2 & R3 & R4 & R5 & R6 & R7 & R8 & R9 & R10 \\
\hline 0.05 & 0.03 & 0.04 & 0.03 & 0.01 & 0.05 & 0.02 & 0.01 & 0.02 & 0.05 \\
\hline
\end{tabular}

Table 3: Sample infectivity probabilities $(f)$, by region (uniform random numbers)

the distance between regions increases, according to the power law function.

\section{The metapopulation Bass diffusion model in $\mathbf{R}$}

The R implementation for the metapopulation Bass model is now presented. First, the relevant libraries are attached, and these include utilities to support plotting (ggplot2 and scales), preparation of data (reshape2 and dplyr), and for performing numerical integration (deSolve).

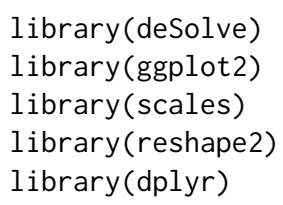

Initially, a data frame is used to specify the model topology, although spatial data stored in GIS files could also be used as part of the implementation (Bivand et al., 2013, pp. 1-16).

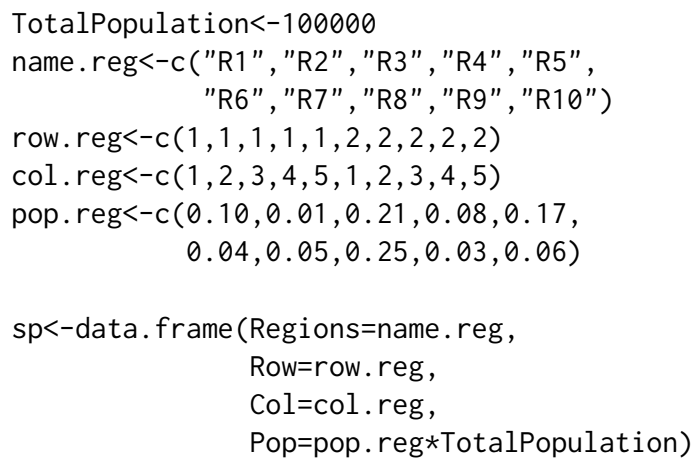

Before running the simulation, the $N x N$ matrix from equation (11) needs be be calculated, based on equations (12), (13), (14), (15) and (16). The normal contact rate is drawn from a random uniform

\begin{tabular}{ccccccccccc}
\hline & R1 & R2 & R3 & R4 & R5 & R6 & R7 & R8 & R9 & R10 \\
\hline R1 & 0.00 & 1.00 & 2.00 & 3.00 & 4.00 & 1.00 & 1.41 & 2.24 & 3.16 & 4.12 \\
R2 & 1.00 & 0.00 & 1.00 & 2.00 & 3.00 & 1.41 & 1.00 & 1.41 & 2.24 & 3.16 \\
R3 & 2.00 & 1.00 & 0.00 & 1.00 & 2.00 & 2.24 & 1.41 & 1.00 & 1.41 & 2.24 \\
R4 & 3.00 & 2.00 & 1.00 & 0.00 & 1.00 & 3.16 & 2.24 & 1.41 & 1.00 & 1.41 \\
R5 & 4.00 & 3.00 & 2.00 & 1.00 & 0.00 & 4.12 & 3.16 & 2.24 & 1.41 & 1.00 \\
R6 & 1.00 & 1.41 & 2.24 & 3.16 & 4.12 & 0.00 & 1.00 & 2.00 & 3.00 & 4.00 \\
R7 & 1.41 & 1.00 & 1.41 & 2.24 & 3.16 & 1.00 & 0.00 & 1.00 & 2.00 & 3.00 \\
R8 & 2.24 & 1.41 & 1.00 & 1.41 & 2.24 & 2.00 & 1.00 & 0.00 & 1.00 & 2.00 \\
R9 & 3.16 & 2.24 & 1.41 & 1.00 & 1.41 & 3.00 & 2.00 & 1.00 & 0.00 & 1.00 \\
R10 & 4.12 & 3.16 & 2.24 & 1.41 & 1.00 & 4.00 & 3.00 & 2.00 & 1.00 & 0.00 \\
\hline
\end{tabular}

Table 4: Regional distance matrix based on Euclidean distance 


\begin{tabular}{ccccccccccc}
\hline & R1 & R2 & R3 & R4 & R5 & R6 & R7 & R8 & R9 & R10 \\
\hline R1 & 4.58 & 0.28 & 3.37 & 1.15 & 2.00 & 0.82 & 1.05 & 4.47 & 0.37 & 0.58 \\
R2 & 2.29 & 0.56 & 5.06 & 1.54 & 2.50 & 0.68 & 1.26 & 5.99 & 0.47 & 0.71 \\
R3 & 1.53 & 0.28 & 10.12 & 2.31 & 3.33 & 0.51 & 1.05 & 7.23 & 0.63 & 0.91 \\
R4 & 1.14 & 0.19 & 5.06 & 4.61 & 5.00 & 0.39 & 0.78 & 5.99 & 0.77 & 1.22 \\
R5 & 0.92 & 0.14 & 3.37 & 2.31 & 10.00 & 0.32 & 0.61 & 4.47 & 0.63 & 1.47 \\
R6 & 2.29 & 0.23 & 3.13 & 1.11 & 1.95 & 1.64 & 1.26 & 4.82 & 0.38 & 0.59 \\
R7 & 1.90 & 0.28 & 4.19 & 1.43 & 2.40 & 0.82 & 2.53 & 7.23 & 0.51 & 0.74 \\
R8 & 1.41 & 0.23 & 5.06 & 1.91 & 3.09 & 0.55 & 1.26 & 14.46 & 0.77 & 0.98 \\
R9 & 1.10 & 0.17 & 4.19 & 2.31 & 4.14 & 0.41 & 0.84 & 7.23 & 1.53 & 1.47 \\
R10 & 0.89 & 0.13 & 3.13 & 1.91 & 5.00 & 0.33 & 0.63 & 4.82 & 0.77 & 2.95 \\
\hline
\end{tabular}

Table 5: Final contact rates $(c)$ from region to region, with $\alpha=1.00$

distribution based on the population proportion in each sector, where areas of higher density will have great interaction rates. The values for infectivity (a measure of the persuasiveness of an adopter) are also drawn from a uniform distribution, and the distance matrix is developed by calling the $\mathrm{R}$ function $\operatorname{dist}()$. The value for $\alpha$ is arbitrarily chosen at 1.00 .

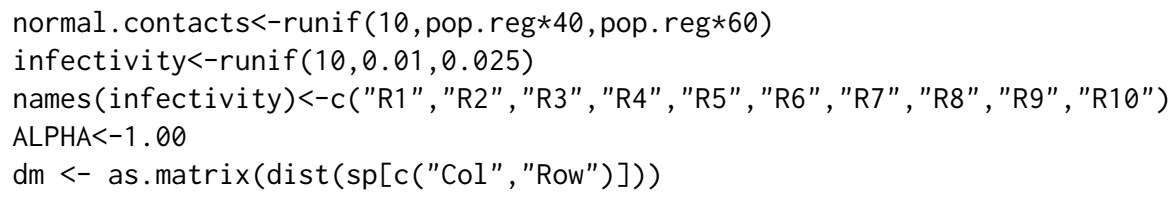

In order to create the $\beta$ matrix, a modified contact rate is calculated based on equation (16), and this is then transformed to an effective contact matrix by multiplying by the infectivity vector. The $\beta$ matrix is then evaluated by simply dividing the effective contacts by the population of each sector, as specified earlier in equation (12).

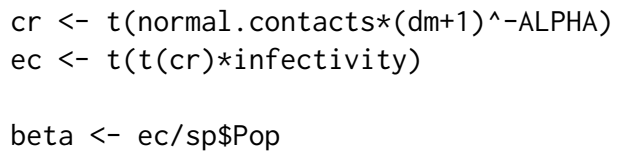

With the contact information defined, the simulation model logic can then be implemented as part of the deSolve package. The key advantage here is that deSolve supports vectorization, and therefore the simulation model is scalable, as it uses matrix algebra to implement (11). Before defining the callback model function, the important simulation parameters are specified, including the start time, finish time, integration time step, the number of regions and the number of integrals (stocks) per region. The simulation time vector is defined, as well as a vector of integrals (20 in all), along with their initial values. For the initial conditions, the initial customer zero adopter is seeded in region eight (an arbitrary choice).

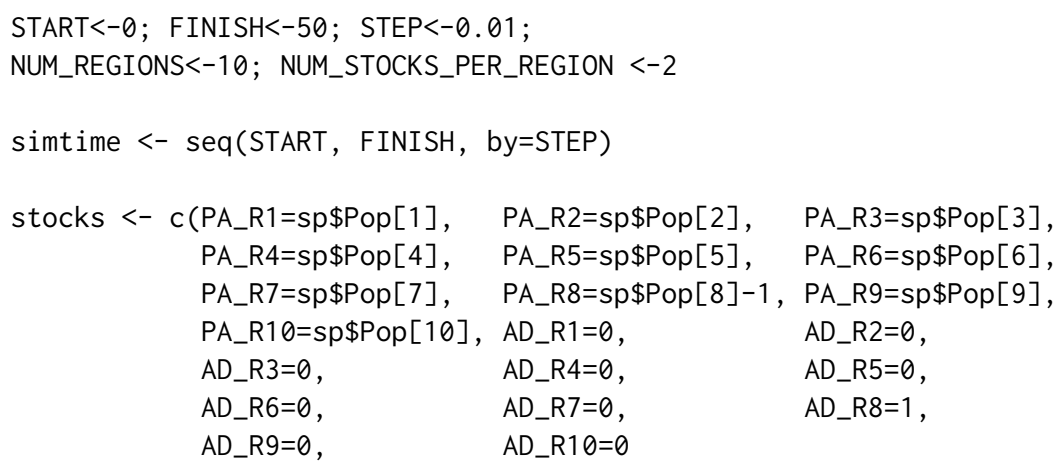

In the model function, the stocks vector is converted into a $10 \times 2$ matrix, where all the potential adopter stocks are in the first column, and the adopter stocks reside in the second column. Matrix algebra is used to calculate the force of persuasion (12), and from this the adoption rates for each sector are calculated (9), followed by the derivatives. Additional information such as the individual adoption 
rates, the total population, the total potential adopters and the total adopters are also returned from the function. A powerful feature of this function is that it is eminently scalable, and would work (assuming availability of sufficient computer memory) on much larger matrices and models.

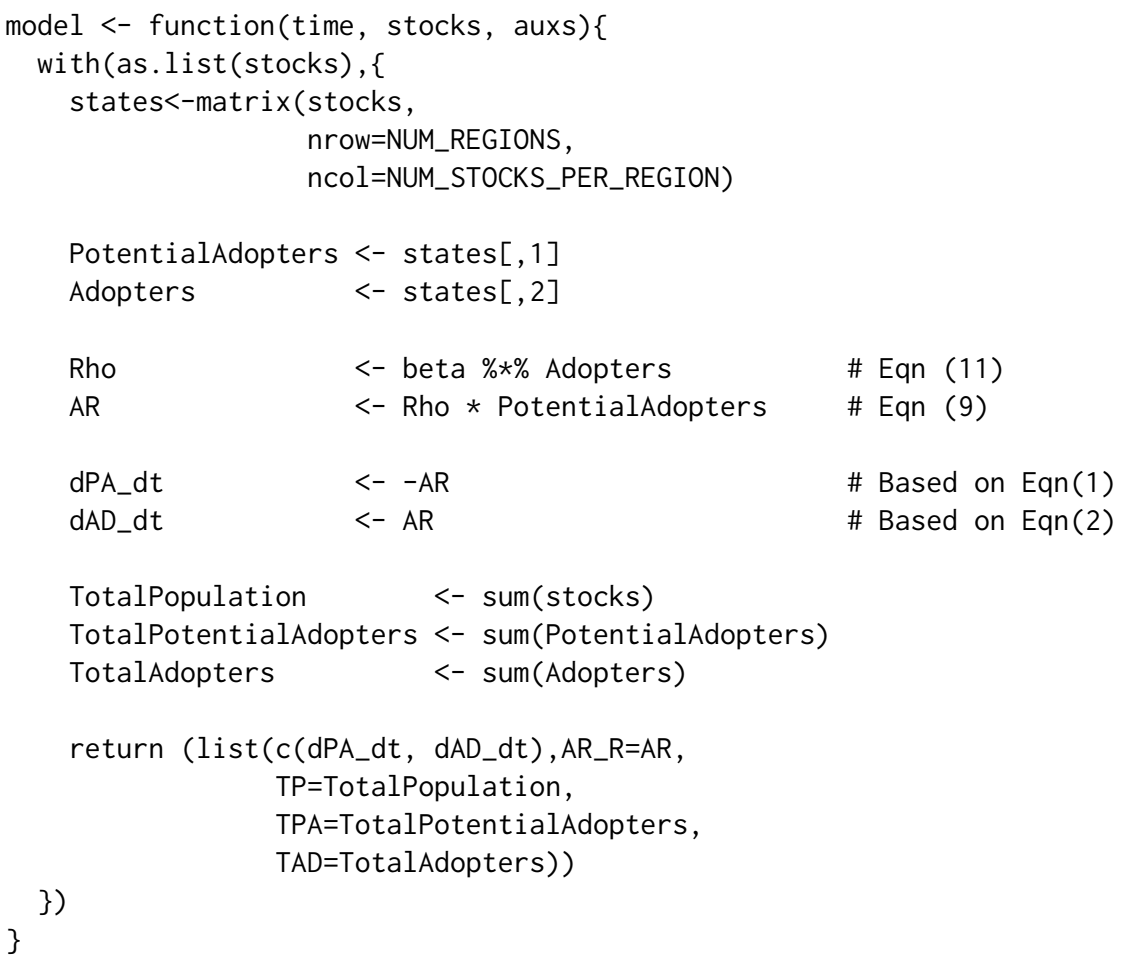

Once the model function has been specified, the deSolve package is called via the ode() function call, as descibed earlier. To prepare the data for visualization, it is filtered to focus on only the simulation data from discrete time steps $(0,1,2, \ldots, 50)$, and this is melted into a three column data frame. The grepl() function is inside the filter() function to extract variables, and these are printed using the function ggplot().

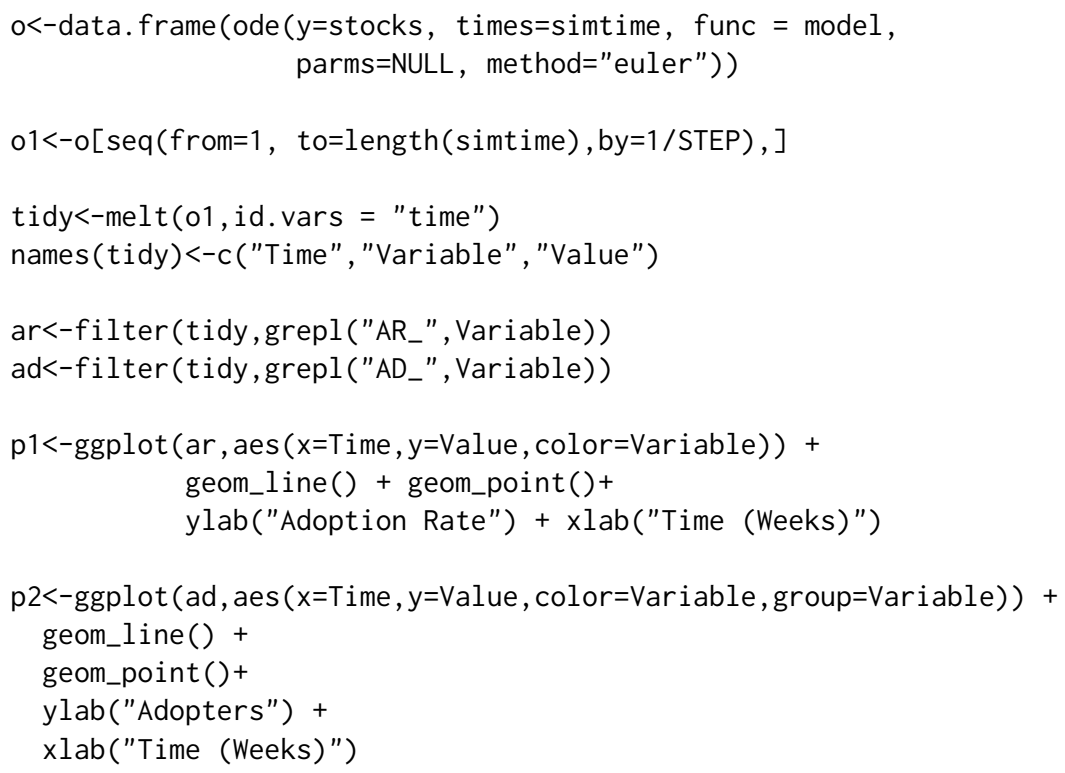

The simulation output is displayed in Figures 4 and 5. The first of these figures shows the adoption rate $(A R)$ values, which follow the classic bell-shaped contagion-like curve, as word of mouth spreads through the population with a correponding increase in the "attack rate" or force of persuasion. As the number of potential adopters decline, so to does the adoption rate. The differences in peak times, although relatively small, are still clear on the diagram, which demonstrates the value of being able to model across spatial regions, and observe how the "epidemic", which is captured in the model equations, spreads across the different regions. The second plot captures the market saturation for each region, as potential adopters convert to adopters via the word of mouth diffusion process. 


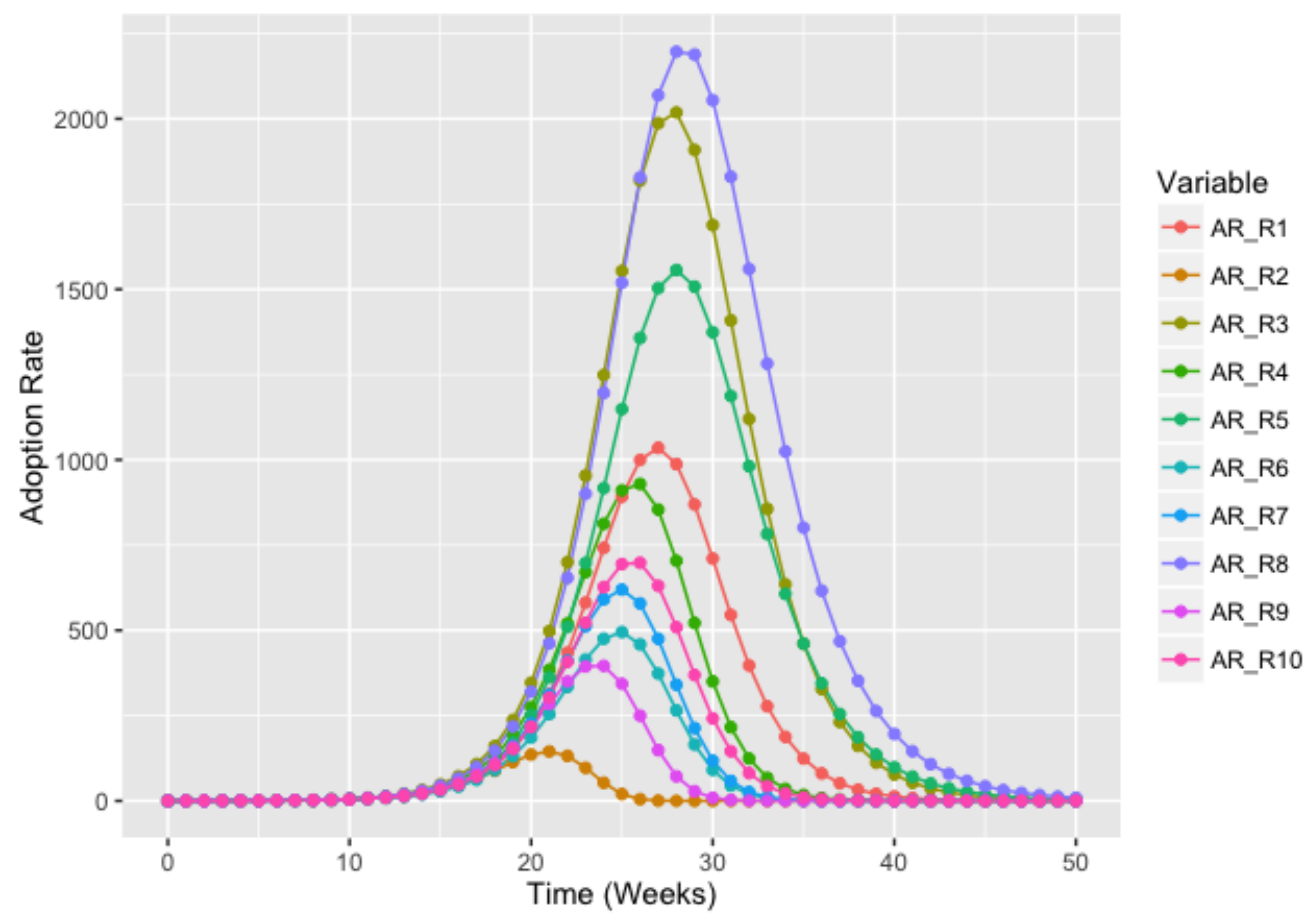

Figure 4: Simulation output: adoption rates by sector

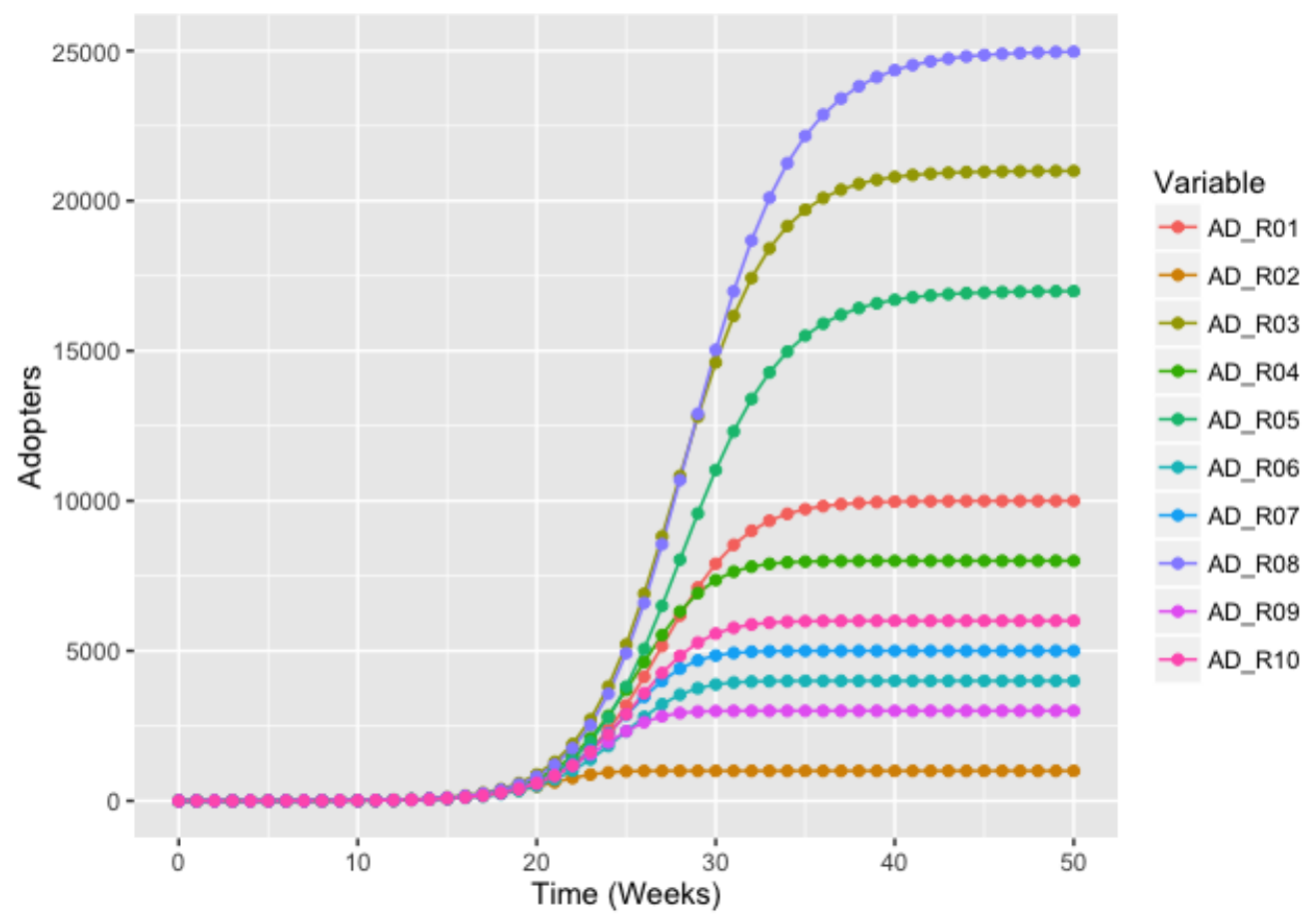

Figure 5: Simulation output: Adopter stocks behavior over time 
While the model is based on hypothetical data, including the regional structure, the contact rates and infectivity, its underlying structure is robust and can be adapted for many spatial interaction processes. The simulation engine of the model is concise and can be used across a number of contexts, and real-world data can be conveniently used within the model, once it is prepared and respresented in matrix format.

\section{Discussion}

This paper shows how $\mathrm{R}$ can be used to model metapopulation integral models of product diffusion. For this class of problems, there are a number of advantages in considering $\mathrm{R}$ as a solution:

- $\mathrm{R}$ provides matrix support, and matrix equations such as the force of persuasion can be easily implemented.

- The package deSolve fully supports vectorisation, which means that the equation writing process is simplified, and the equation structures developed are scalable without having to alter the underlying model structure.

- $\mathrm{R}$ also provides excellent statistical analysis support, and model analysis techniques such as statistical screening (Ford and Flynn, 2005) can be conveniently scripted using R Duggan (2016, chapter 7).

Further extensions to the work would be to apply optimization techniques in order to fit product diffusion models to product sales data across regions, and therefore obtain more realistic simulations of the peak time (Vynnycky and Edmunds, 2008). These model extensions could also accomodate age cohort dynamics for market-based models, for example, those captured in epidemiological models such as influenza transmission (Keeling and Rohani, 2008; Vynnycky and White, 2010).

\section{Bibliography}

R. M. Anderson, R. M. May, and B. Anderson. Infectious Diseases of Humans: Dynamics and Control. Oxford University Press, Oxford, UK., 1991. [p154]

O. S. Baquero and F. S. Marques. EpiDynamics: Dynamic Models in Epidemiology, 2015. URL https: //cran.r-project.org/package=EpiDynamics. R package version 0.3.0. [p155]

F. M. Bass. A new product growth for model consumer durables. Management Science, 15(5):215-227, 1969. URL https://doi.org/10.1287/mnsc.15.5.215. [p153]

R. S. Bivand, E. Pebesma, and V. Gómez-Rubio. Applied Spatial Data Analysis with R. Springer-Verlag, New York, NY, 2013. [p158]

M. Borgdorff, N. Nagelkerke, D. Van Soolingen, and J. Broekmans. Transmission of tuberculosis between people of different ages in the netherlands: An analysis using dna fingerprinting. The International Journal of Tuberculosis and Lung Disease, 3(3):202-206, 1999. [p156]

D. Brockmann, L. Hufnagel, and T. Geisel. The scaling laws of human travel. Nature, 439(7075):462-465, 2006. [p157]

J. Duggan. System Dynamics Modeling with R. Springer-Verlag, Switzerland, 2016. URL https: //doi.org/10.1007/978-3-319-34043-2. [p154,162]

A. Ford and H. Flynn. Statistical screening of system dynamics models. System Dynamics Review, 21(4): 273-303, 2005. URL https://doi.org/10.1002/sdr. 322. [p162]

S. Jenness, S. M. Goodreau, M. Morris, E. Beylerian, S. Bender-deMoll, and K. Weiss. EpiModel: Mathematical Modeling of Infectious Disease, 2016. URL https://cran.r-project.org/package= EpiModel. R package version 1.2.8. [p155]

M. J. Keeling and P. Rohani. Modeling Infectious Diseases in Humans and Animals. Princeton University Press, Princeton, New Jersey 08540, 2008. [p156, 162]

D. C. Lane. IFORS operational research Hall of Fame Jay Wright Forrester. International Transactions in Operational Research, 13(5):483-492, 2006. [p154]

X. Li and C. Prasad. Effectively teaching coding standards in programming. In Proceedings of the 6th Conference on Information Technology Education, pages 239-244. ACM, 2005. [p155] 
H. Liu, E. Howley, and J. Duggan. Co-evolutionary analysis: a policy exploration method for system dynamics models. System Dynamics Review, 28(4):361-369, 2012. URL https: //doi . org/10.1002/ sdr. 1482. [p157]

S. Meyer, L. Held, and others. Power-law models for infectious disease spread. The Annals of Applied Statistics, 8(3):1612-1639, 2014. URL https://doi .org/10.1214/14-aoas743. [p157]

J. Mossong, N. Hens, M. Jit, P. Beutels, K. Auranen, R. Mikolajczyk, M. Massari, S. Salmaso, G. S. Tomba, J. Wallinga, and others. Social contacts and mixing patterns relevant to the spread of infectious diseases. PLoS Med, 5(3):e74, 2008. URL https: //doi.org/10.1371/journal. pmed.0050074. [p156]

D. Mungovan, E. Howley, and J. Duggan. The influence of random interactions and decision heuristics on norm evolution in social networks. Computational and Mathematical Organization Theory, 17(2): 152-178, 2011. URL https://doi.org/10.1007/s10588-011-9085-7. [p157]

J. A. Norton and F. M. Bass. A diffusion theory model of adoption and substitution for successive generations of high-technology products. Management science, 33(9):1069-1086, 1987. URL https: //doi.org/10.1287/mnsc.33.9.1069. [p153]

H. Rahmandad and J. Sterman. Heterogeneity and network structure in the dynamics of diffusion: Comparing agent-based and differential equation models. Management Science, 54(5):998-1014, 2008. [p153]

K. Soetaert, T. Petzoldt, and R. W. Setzer. Solving Differential Equations in R: Package deSolve. Journal of Statistical Software, 33, 2010. [p155]

K. Soetaert, T. Petzoldt, and R. W. Setzer. deSolve: Solvers for Initial Value Problems of Differential Equations (ODE, DAE, DDE), 2016. URL https://cran. r-project.org/package=deSolve. R package version 1.14. [p155]

J. D. Sterman. Business Dynamics: Systems Thinking and Modeling for a Complex World, volume 19. Irwin/McGraw-Hill Boston, New York, 2000. [p154, 155]

E. Vynnycky and W. Edmunds. Analyses of the 1957 (Asian) Influenza Pandemic in the United Kingdom and the Impact of School Closures. Epidemiology and infection, 136(2):166-179, 2008. [p162]

E. Vynnycky and R. White. An Introduction to Infectious Disease Modelling. OUP Oxford, 2010. [p153, 162]

Jim Duggan

School of Engineering and Informatics,

National University of Ireland Galway,

Galway,

Ireland.

jim.duggan@nuigalway.ie 